\title{
Consciousness operates beyond the timescale for discerning time intervals: implications for $Q$-mind theories and analysis of quantum decoherence in brain
}

\author{
Danko Dimchev Georgiev ${ }^{1,2}$ \\ ${ }^{1}$ Division of Electron Microscopy, Medical University of Varna, Bulgaria \\ ${ }^{2}$ Department of Emergency Medicine, Bregalnitsa Street 3, Varna, Bulgaria
}

This paper presents in details how the subjective time is constructed by the brain cortex via reading packets of information called "time labels", produced by the right basal ganglia that act as brain timekeeper. Psychophysiological experiments have measured the subjective "time quanta" to be $40 \mathrm{~ms}$ and show that consciousness operates beyond that scale - an important result having profound implications for the Q-mind theory. Although in most current mainstream biophysics research on cognitive processes, the brain is modelled as a neural network obeying classical physics, Penrose $(1989,1997)$ and others have argued that quantum mechanics may play an essential role, and that successful brain simulations can only be performed with a quantum computer. Tegmark (2000) showed that make-or-break issue for the quantum models of mind is whether the relevant degrees of freedom of the brain can be sufficiently isolated to retain their quantum coherence and tried to settle the issue with detailed calculations of the relevant decoherence rates. He concluded that the mind is classical rather than quantum system, however his reasoning is based on biological inconsistency. Here we present detailed exposition of molecular neurobiology and define the dynamical timescale of cognitive processes linked to consciousness to be 10-15 picoseconds showing that macroscopic quantum coherent phenomena in brain are not ruled out, and even may provide insight in understanding life, information and consciousness.

\section{The brain cortex hosts our mind}

The brain cortex is the main residence of consciousness. All sensory stimuli are realized only when they reach the brain cortex and not before that! Nieuwenhuys (1994) outlines the origin and evolutionary development of the neocortex. A cortical formation is lacking in amphibians, but a simple three-layered cortex is present throughout the pallium of reptiles. In mammals, two three-layered cortical structures, i.e. the prepiriform cortex and the hippocampus, are separated from each other by a six-layered neocortex. Still small in marsupials and insectivores, this new structure attains amazing dimensions in anthropoids and cetaceans. Neocortical neurons can be allocated to one of two basic categories: pyramidal and nonpyramidal cells. The pyramidal neurons form the principal elements in neocortical circuitry, accounting for at least $70 \%$ of the total neocortical population. The evolutionary development of the pyramidal neurons can be traced from simple, "extraverted" neurons in the amphibian pallium, via pyramid-like neurons in the reptilian cortex to the fully developed neocortical elements designated by Cajal as psychic cells. 
Typical mammalian pyramidal neurons have the following eight features in common: (1) spiny dendrites, (2) a stout radially oriented apical dendrite, forming (3) a terminal bouquet in the most superficial cortical layer, (4) a set of basal dendrites, (5) an axon descending to the subcortical white matter, (6) a number of intracortical axon collaterals, (7) terminals establishing synaptic contacts of the round vesicle/asymmetric variety, and (8) the use of the excitatory aminoacids glutamate and/or aspartate as their neurotransmitter. The pyramidal neurons constitute the sole output and the largest input system of the neocortex. They form the principal targets of the axon collaterals of other pyramidal neurons, as well as of the endings of the main axons of cortico-cortical neurons. Indeed, the pyramidal neurons constitute together a continuous network extending over the entire neocortex, justifying the generalization: the neocortex communicates first and foremost within itself!

The typical pyramidal neurons represent the end stage of a progressive evolutionary process. During further development many of these elements have become transformed by reduction into various kinds of atypical or aberrant pyramidal neurons. Interestingly, none of the six morphological characteristics, mentioned above under 1-6, has appeared to be unassailable; pyramidal neurons lacking spines, apical dendrites, long axons and intracortical axon collaterals etc. have all been described. From an evolutionary point of view the typical pyramidal neurons represent not only the principal neocortical elements, but also the source of various excitatory local circuit neurons. The spiny stellate cells, which are abundant in highly specialized primary sensory areas, form a remarkable case in point.

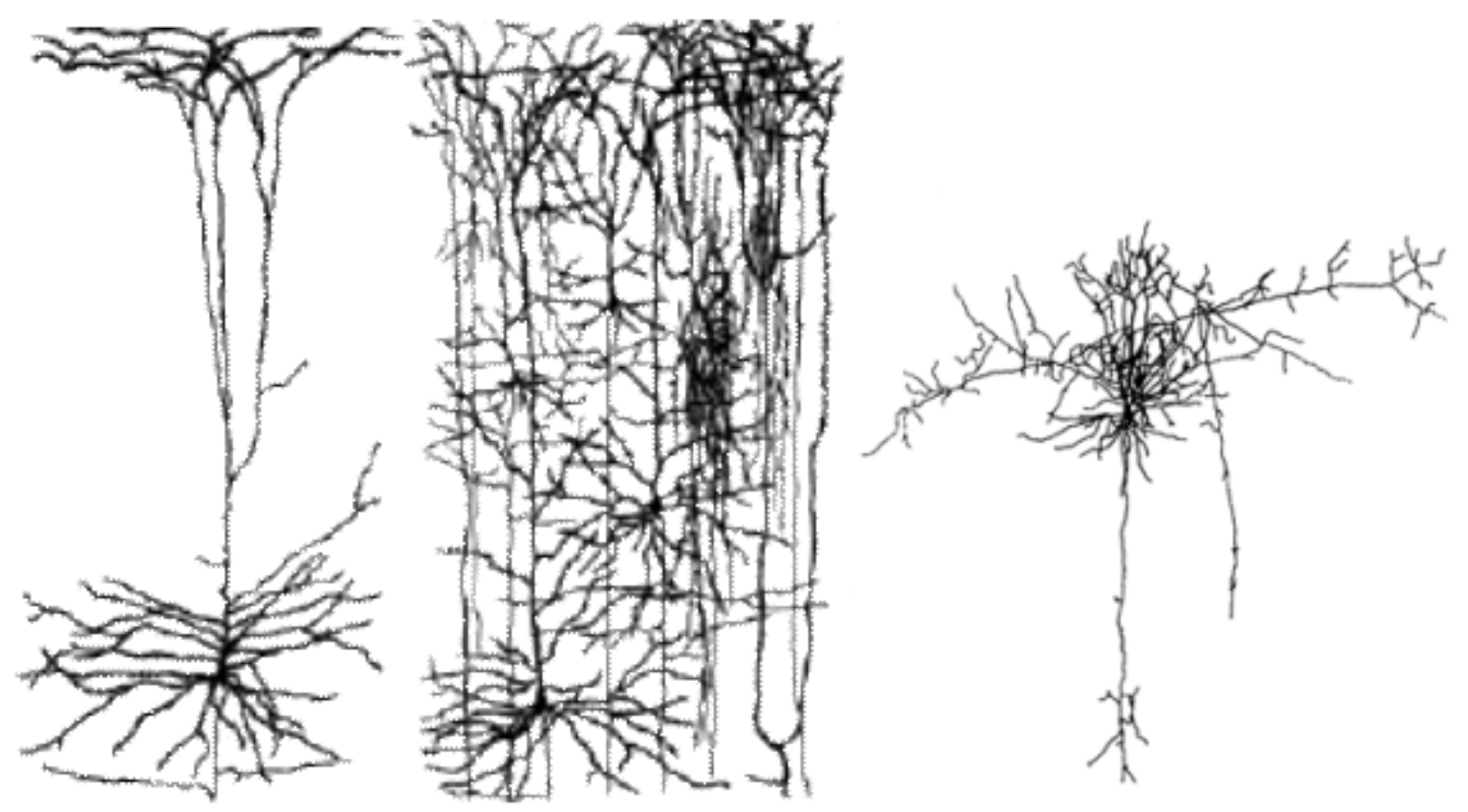

FIG.1 Types of neocortical neurons. Left: Deep principal neocortical pyramidal neuron. Middle: Superficial principal neocortical pyramidal neurons. Right: Neocortical basket cell that is inhibitory GABA operating interneuron. 
In the clinical neurology the main doctrine says that each side of the body is controlled by the opposite side of the brain cortex that is why the sensory information must also be conveyed to the opposite side of the brain cortex, so that it "knows" the current status of the structures it is supposed to move. Axons of primary sensory neurons are uncrossed; they synapse on secondary sensory neurons located on the same side of the CNS. Most of the information coming to the cerebral cortex is first processed by the thalamus, and relayed in thalamocortical fibers. The cortex outputs information back to the thalamus via corticothalamic fibers. Almost all thalamocortical and corticothalamic fibers are uncrossed. Because all sensory information from one side of the body eventually reaches the opposite cerebral cortex this means that crossings in each of the sensory systems must occur between the secondary sensory neurons, wherever they are, and the thalamus.

Here we will discuss the organization of the sensory pathway of pain. This sensory pathway is composed from three relay neurons. The body of the first sensory neurons is located in the dorsal root ganglion, the body of the second neuron is located in lamina I, II or V of the spinal cord dorsal horn and the third neuron is located in the thalamus. The axon of the dorsal root ganglion neuron is branched: the peripheral part is indeed part of the sensory nerves, while the central part terminates on neurons located in the lamina I, II or V of the spinal cord. The lamina I, II, V spinal neurons extend axons that terminate on thalamic neurons. These axons are part from the spinothalamic tract and cross within the spinal cord. The third neuron from the thalamus delivers information to the brain cortex where the pain is consciously realized. In order to be consciously realized the information travels along the sensory pathway in the form of electric impulses that is why there is delay time before the peripheral reception of the stimulus and conscious experience.

The sensory pathways for touch, vibration, temperature, spatial orientation etc. have analogous anatomical organization that is composed from three relay neurons. The special senses like vision and hearing have more complex organization and up to six relay neurons before the information enters the brain cortex where it is consciously realized. The clinical practice has shown that the brain cortex is the only conscious structure, while all the electric impulses in structures subserving the cortex (like thalamus, basal ganglia or cerebellum) remain unconscious. Thus there is perfect matching between conscious/unconscious process and cortical/noncortical processed information. In order to elucidate this let us consider clinical case in which the visual cortex of an adult subject is damaged, but his eyes are healthy. This condition is known as cortical blindness (amaurosis corticalis) in which the patient is blind because the part of the cortex responsible for conscious visual sensations is damaged. Because the patient has intact eyes and intact subcortical structures, the visual information reaches the lateral geniculate nucleus (LGN) and thalamus that is why the pupillar and other types of visual reflexes are preserved. The patient however insists that he could not see anything. This confirms the idea that the impulse must reach the brain cortex in order to be consciously realized; otherwise it would be consciously realized as soon as it reaches the thalamus. 


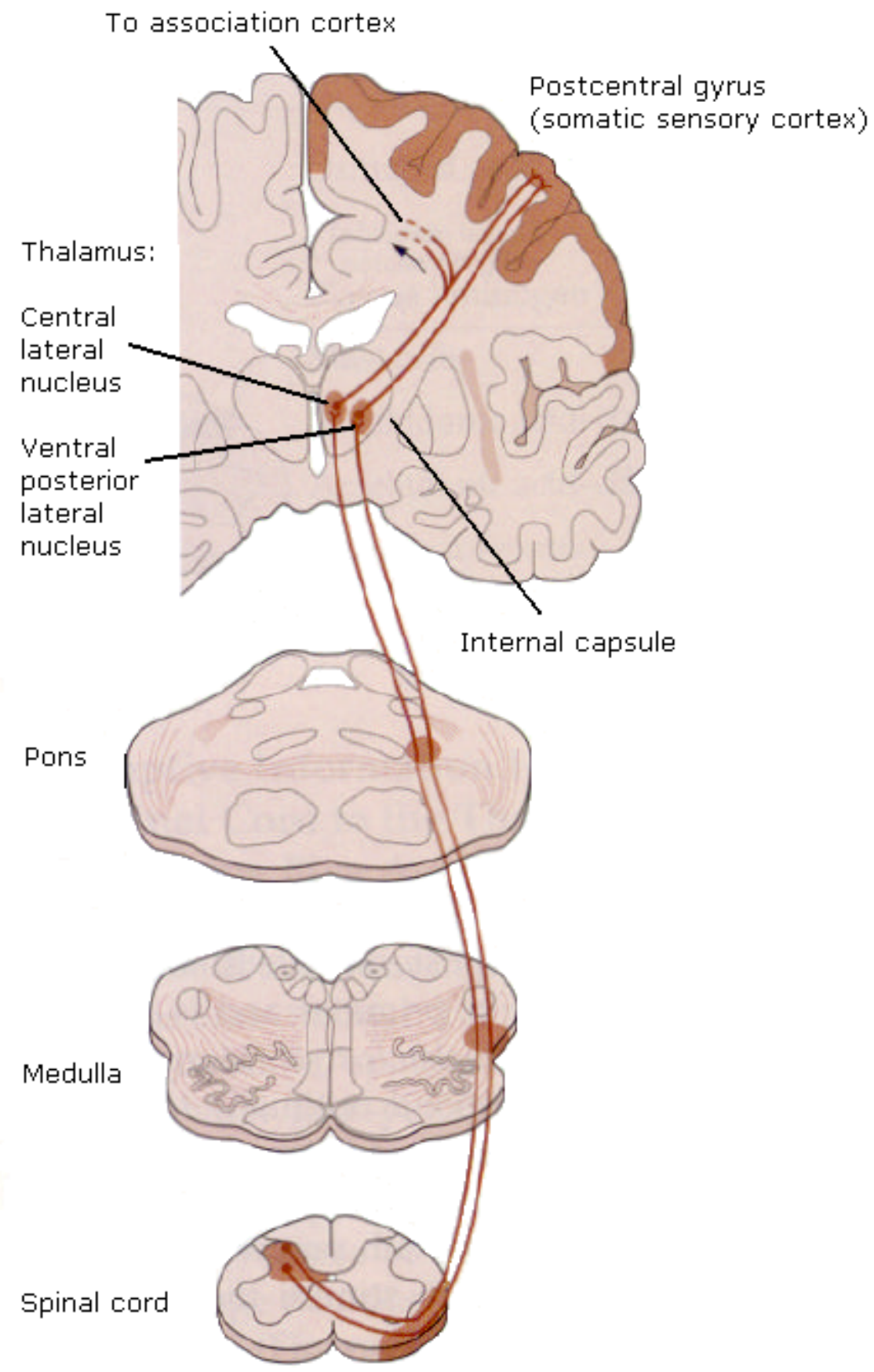

FIG.2 Localization of second and third relay neurons that transmit the neural impulse before pain is realized in the brain cortex. The dorsal root ganglion (first neuron) is not shown on the picture. Spinal cord, medulla and pons are represented with their transversal sections, while thalamus and cortex are shown in frontal slice. 
In order to test the cortical significance in hosting our mind Penfield (1954a, 1954b, 1955) has performed a series of experiments in which he applied weak electric currents in awake patients undergoing brain surgery (craniotomy). He has shown that if certain areas of the brain are exposed and gently stimulated by an electrode, the subject may suddenly be transported in a fully conscious state into some past experience which is recalled with such vividness that he does not seem to himself to be merely remembering but rather to be experiencing all over again the original occasion. The re-lived experience thus produced stops mid-stream, as it were, shortly after the electrode is withdrawn (Penfield \& Perot, 1963). The curious thing is that if the electrode is again contacted near the original site the experience is often re-lived all over again as a kind of re-run. There is not a continuation where the last scene finished off, but a repeat performance. In one subject this occurred 62 successive times! This seems to indicate a rather precise localization within the cortex, like setting the needle down in the same spot on a record. Disconcertingly, however, it is often quite otherwise. One subject, stimulated in the same area, had four apparently unrelated experiential responses. First he heard "footsteps"; secondly, "a company of people in the room"; thirdly, "like being in a gymnasium," and finally "a lady talking to a child at the seashore" (Penfield \& Perot, 1963). But in the case of repetitious recall, nothing has been lost, nor has anything been added. Penfield (1969) said, "Events are not a bit fancifully elaborated as dreams are apt to be when recalled". Nothing whatever is added provided the episode is the same one. The curious thing is that in some of Penfield's experiments, indeed in many of them, the individual was able consciously to identify the meaning of the relived experience, not as a kind of hallucination but as something as real as life, from which he nevertheless stood apart. A woman listening to an orchestra under Penfield's stimulating electrode, hummed the tune she heard, verse and chorus, thus accompanying by an act of conscious effort the very music which was somehow being recalled from the subconscious so vividly. Furthermore, such recallings were entirely involuntary. They are not memories voluntarily brought to the surface. They are more detailed and more vivid than such memories ever are. Penfield reported the experience of one patient who re-experienced an occasion upon which she was sitting in a room and listening to the children playing outside. The sounds of motor traffic and all the other noises of urban living provided the "natural" background. She discussed all this with Dr. Penfield while it was happening, and so real was the experience that it took some time to convince her afterward that he had not actually arranged the whole thing, including the noises outside at the time. Needless to say, he had not done so: it was entirely a vividly re-lived experience complete with all sound effects. It should be noted that when experience is recalled by this technique (via electrode stimulation), the individual is not aware of any process of recall. Only afterwards, at the conclusion of the experiment, is it recognized as a vivid memory from the past. Another patient suddenly found herself sitting on the right hand rear seat of a car, with the window slightly down, waiting at a level crossing for a train to pass. She could even count the carriages as they went by, and all the characteristic sounds and noises were there, complete. After the train had passed and they crossed the tracks into town, even the old familiar smells were experienced. Penfield (1969) says this was the only case of a re-experienced smell that he came across in over a thousand patients whose brain surface was exposed in this way, in an effort to locate the cause of epileptic attacks. Penfield from the evidence believed that the memory record continues intact in the person's mind even after his ability to recall it has disappeared. More than this, he found that if the cortical area, which had been the site of 
stimulation for the re-living of some experience, was subsequently operatively removed (when it was believed to be for the benefit of the epileptic patient), the patient could still voluntarily recall the experience afterwards. Evidently, therefore, the memory itself was not stored at this point, but in some area to which the site was connected. Severing connections to the area made it impossible to obtain recall by electrical stimulation, but it did not eradicate the memory itself, which could therefore still be recalled voluntarily.

The most amazing surgical intervention however was performed by Dobelle (2000), who has succeeded in implanting electrodes in the visual cortex of a blind patient and has helped him to see again using "bionic eyes" (that is a tiny television camera mounted on a pair of glasses). Although the patient called Jerry does not "see" in the conventional sense, he can make out the outlines of objects, large letters and numbers on a contrasting background, and can use the direct digital input to operate a computer. Jerry has been blind since age 36 after a blow to the head. Now 65, he volunteered for the study and got a brain implant in 1978. There has been no infection or rejection in the past 25 years.

Scientists have been working since 1978 to develop and improve the software that enables Jerry to use the device as a primitive visual system. Jerry's "eye" consists of a tiny television camera and an ultrasonic distance sensor mounted on a pair of eyeglasses. Both devices communicate with a small computer, carried on his hip, which highlights the edges between light and dark areas in the camera image. It then tells an adjacent computer to send appropriate signals to an array of 68 small platinum electrodes on the surface of Jerry's brain, through wires entering his skull behind his right ear. The electrodes stimulate certain brain cells, making Jerry perceive dots of light, which are known as phosphenes. Jerry gets white phosphenes on a black background. With small numbers of phosphenes you have (the equivalent of) a time and temperature sign at a bank. As you get larger and larger numbers of phosphenes, you go up to having a sports stadium scoreboard. If he is walking down a hall, the doorway appears as a white frame on a dark background. Jerry demonstrated by walking across a room to pull a woolly hat off a wall where it had been taped, took a few steps to a mannequin and correctly put the hat on its head. A reproduction of what Jerry sees showed crosses on a video screen that changed from black to white when the edge of an object passed behind them on the screen. Jerry can read two-inch tall letters at a distance of five feet. And he can use a computer, thanks to some input from his 8-year-old son, Marty.

The system works by detecting the edges of objects or letters. "When an object passes by the television camera ... I see dots of light. Or when I pass by it," Jerry says. Jerry, currently the only user of the latest system, must move his head slightly to scan what he is looking at. He has the equivalent of 20/400 vision - about the same as a severely nearsighted person - in a narrow field. Although the relatively small electrode array produces tunnel vision, the patient is also able to navigate in unfamiliar environments including the New York City subway system. One other patient who has tried the new system cannot "see" anything with it. According to Dr. Dobelle the man was blinded at the age of 5, 60 years ago, and his brain may have "forgotten" how to use its visual cortex. Also it is not known whether the system will work for people who were born blind. 
All the current medical evidence points out that the human mind is located in the brain cortex. Following the current neural network paradigm it is considered that the neural activity of the brain cortex coincides with the term consciousness, while all extra-cortical activity is not conscious and coincides with the term subconsciousness. Here we will slightly revisit and sharpen the boundaries of mind/consciousness suggesting that is located in intraneuronally organized protein network called cytoskeleton. The cytoskeletons of all cortical neurons are organized in functional unity via intrasynaptic protein-protein bridges connecting the presynaptic and the postsynaptic cytoskeletons. These adhesive protein molecules ( $\beta$-neurexin and neuroligin-1) transmit information in both directions and regulate the exocytosis (neuromediator release) via signals from the cytoskeleton. The proposed sharpening of the boundaries of consciousness and localizing it in coherently organized brain cortical cytoskeletons is not fully incompatible with the current neural network paradigm indeed it extends it, showing input of information from the membrane electric activity down to the cytoskeleton and effective feedback regulation of the neuronal electric activity by the cytoskeleton.

\section{The neuronal cytoskeleton and its link to cognition}

With the development of molecular biology and molecular psychiatry it became clear that the neurons are highly complex and the cognitive processes could be understood only if studying the molecular changes taking part in memory formation, addiction, psychiatric disorders like schizophrenia, Alzheimer's disease, different types of dementias, bipolar disorder etc. Surprisingly it was shown that in all these instances there are changes in the neuromediator activity, neuronal firing and the intraneuronal cytoskeletal organization. Considering that the proteins are the essential biomolecules operating in picosecond timescales and performing specific functions responsible for emergence and sustaining of life it is reasonable to conclude that the changes in neuromediator activity and neuronal firing are secondary to the cytoskeletal (intra-neuronal and synaptic) reorganization. Indeed many details of the proposed cytoskeletal regulation of synaptic structure and exocytosis are already known.

In our further discussion we will not take into consideration the changes in the gene expression, assuming that the transfer of information between the cytoskeleton and the cell nucleus subserves the needs of the cytoskeleton, the organization of the neuronal plasma membrane and the membrane bound compartments. The genetic material (DNA and RNA) is considered as set of fundamental information essential for cell functioning and life, but not directly linked to consciousness, which is something dynamic that develops in time from childhood to adolescence and progressively regresses with advance of the senile changes. In the next several sections we will discuss in details the cytoskeletal involvement in different types of cognitive processes and psychiatric disorders. 


\section{GPCRs, cytoskeleton and psychiatric disorders}

Interneuronal communication at chemical synapses is achieved via release of neuromediator molecules from the presynaptic terminals that act on postsynaptic membrane bound receptors. The postsynaptic receptors could be divided in two major classes: ionotropic and metabotropic. The ionotropic receptors are indeed channels that increase their conductance for ions $\left(\mathrm{Na}^{+}, \mathrm{K}^{+}, \mathrm{Ca}^{2+}, \mathrm{Cl}^{-}, \mathrm{HCO}_{3}^{-}\right)$when the neuromediator molecule (ligand) is bound to specific regulatory place (usually "pocket") on the channel. This is just half-the-story considered by the neural network theorists. The neuromediators bind also to metabotropic receptors called G-protein coupled receptors (GPCRs) or 7-TM receptors (because they consist of 7 transmembrane $\alpha$-helices). CPCRs activate second messengers inside the neurons that could activate different protein kinases and phosphatases that modulate the cytoskeletal activity. The cytoskeleton then reorganizes the neuronal shape, the synaptic morphology, sends signals to the nucleus in order to cover its need of plastic ingredients (yields synthesis of mRNA that encodes the primary aminoacid sequence of the proteins needed).

Table I. Basic neurotransmitters and neurotransmitter receptors in brain ${ }^{*}$

\begin{tabular}{|c|c|c|}
\hline Neurotransmitter & Receptors & Type \\
\hline \multirow[t]{2}{*}{ Dopamine } & $\mathrm{D}_{1}, \mathrm{D}_{5}$ & $\mathrm{G}_{\mathrm{s}}$ \\
\hline & $\mathrm{D}_{2}, \mathrm{D}_{3}, \mathrm{D}_{4}$ & $\mathrm{G}_{\mathrm{i} / \mathrm{o}}$ \\
\hline \multirow[t]{4}{*}{ Serotonin } & $5-\mathrm{HT}_{1}$ & $\mathrm{G}_{\mathrm{i} / \mathrm{o}}$ \\
\hline & $5-\mathrm{HT}_{2}$ & $\mathrm{G}_{\mathrm{q} / 11}$ \\
\hline & $5-\mathrm{HT}_{3}$ & ion channel \\
\hline & $5-\mathrm{HT}_{4}, 5-\mathrm{ht}_{5}, 5-\mathrm{ht}_{6}, 5-\mathrm{HT}_{7}$ & $\mathrm{G}_{\mathrm{s}}$ \\
\hline \multirow{3}{*}{ Norepinephrin } & $\alpha_{1}$ & $\mathrm{G}_{\mathrm{q} / 11}$ \\
\hline & $\alpha_{2}$ & $\mathrm{G}_{\mathrm{i} / \mathrm{o}}$ \\
\hline & $\beta_{1}, \beta_{2}, \beta_{3}$ & $\mathrm{G}_{\mathrm{s}}$ \\
\hline \multirow[t]{3}{*}{ Acetylcholine } & $\mathrm{M}_{1}, \mathrm{M}_{3}, \mathrm{M}_{5}$ & $\mathrm{G}_{\mathrm{q} / 11}$ \\
\hline & $\mathrm{M}_{2}, \mathrm{M}_{4}$ & $\mathrm{G}_{\mathrm{i} / \mathrm{o}}$ \\
\hline & $\alpha_{1}^{*}, \alpha_{2}{ }^{*}, \alpha_{3}^{*}, \alpha_{4}^{*}, \alpha_{6}^{*}, \alpha_{7}, \alpha_{7}^{*}, \alpha_{8}, \alpha_{8}^{*}, \alpha_{9}, \alpha_{9}^{*}, \alpha_{10}{ }^{*}$ & ion channels \\
\hline Opioid peptides & $\mu, \kappa, \delta, \mathrm{N} / \mathrm{OFQ}$ & $\mathrm{G}_{\mathrm{i} / \mathrm{o}}$ \\
\hline Cannabinoids & $\mathrm{CB}_{1}, \mathrm{CB}_{2}$ & $\mathrm{G}_{\mathrm{i} / \mathrm{o}}$ \\
\hline \multirow[t]{2}{*}{$G A B A$} & $\mathrm{GABA}_{\mathrm{A}}, \mathrm{GABA}_{\mathrm{C}}$ & ion channels \\
\hline & $\mathrm{GABA}_{\mathrm{B}}$ & $\mathrm{G}_{\mathrm{i} / 0}$ \\
\hline \multirow[t]{4}{*}{ Glutamate } & NMDA, AMPA, kainate & ion channels \\
\hline & $\mathrm{mglu}_{1}, \mathrm{mglu}_{5}$ & $\mathrm{G}_{\mathrm{q} / 11}$ \\
\hline & $\mathrm{mglu}_{2}, \mathrm{mglu}_{3}$ & $\mathrm{G}_{\mathrm{i} / \mathrm{o}}$ \\
\hline & $\mathrm{mglu}_{4}, \mathrm{mglu}_{6}, \mathrm{mglu}_{7}, \mathrm{mglu}_{8}$ & $\mathrm{G}_{\mathrm{i} / \mathrm{o}}$ \\
\hline
\end{tabular}

\footnotetext{
* The metabotropic receptors are annotated with the associated G-proteins, while the ionotropic receptors are annotated as ion channels.
} 
The G-proteins are heterotrimers composed from $\alpha, \beta$ and $\gamma$ subunit. There are 4 main classes of G-proteins depending on the $\alpha$-subunit type. $G_{s}$ proteins have $\alpha_{\mathrm{s}}$ or $\alpha_{\text {olf }}$ subunits. The $\alpha_{\mathrm{s}}$ subunit stimulates adenylyl cyclase that increases the level of intracellular second messenger cAMP, which activates protein kinase A (PKA). The $\alpha_{\text {olf }}$ subunit stimulates the adenylyl cyclase in peripheral olfactory neurons. $G_{i}$ proteins possess 4 types of $\alpha$-subunits: $\alpha_{i 1}$ inhibits the adenylyl cyclase, $\alpha_{\mathrm{i} 2}$ opens $\mathrm{K}^{+}$channels, $\alpha_{0}$ inhibits adenylyl cyclase I, opens $\mathrm{K}^{+}$channels and closes $\mathrm{Ca}^{2+}$ channels, $\alpha_{\mathrm{t}}$ called transducin is present in rods and cones and activates cGMP phosphodiesterase in the cascade of photon perception. $G_{q}$ proteins have 4 types of $\alpha$-subunits: $\alpha_{\mathrm{q}}$ activates phospholipase C $\beta_{1}\left(\mathrm{PLC} \beta_{1}\right), \alpha_{11}$ activates PLC $\beta_{2}, \alpha_{14}$ and $\alpha_{16}$ activate PLC $\beta_{1}$ and PLC $\beta_{3}$. $G_{12}$ proteins possess $\alpha_{12}$ or $\alpha_{13}$ subunits and activate phospholipase $\mathrm{A}_{2}$ $\left(\mathrm{PLA}_{2}\right) . \mathrm{G} \alpha$ subunits are shown also to interact with microtubules, while the $\beta \gamma$ subunit interacts with GDP-bound tubulin and promotes microtubule assembly.

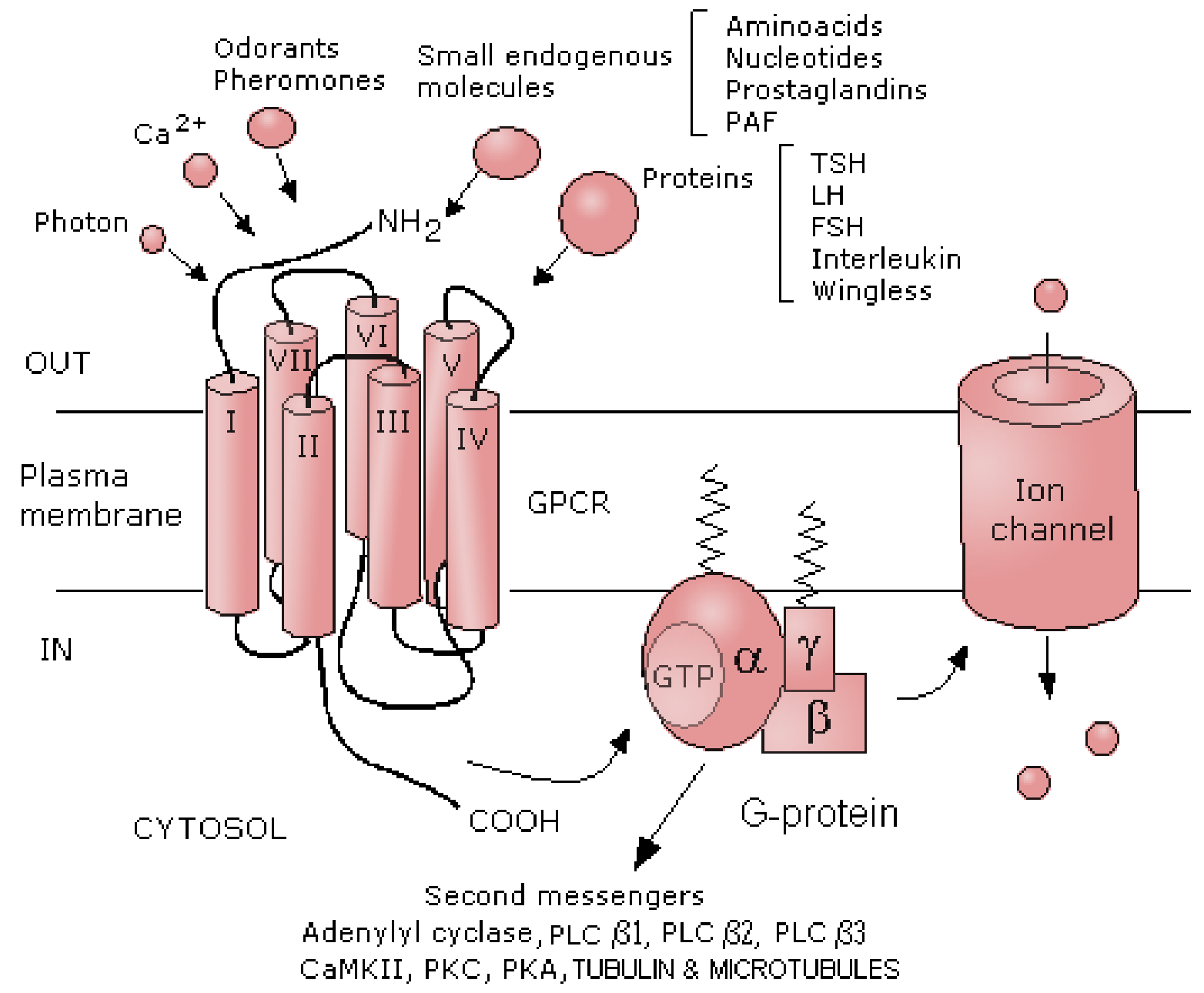

FIG.3 Two basic types of plasmalemmal receptors: ionotropic and metabotropic (GPCRs). The picture shows some of the GPCR ligands and the G-protein mediated activation of different intracellular enzymes. 
In psychiatry almost all known disorders are consequence of disregulated GPCR function, disregulation of the neurotransmitter systems, and associated alteration of the neuronal cytoskeletons that leads to morphologic changes of the neurites (dendrites and axons) and the synaptic structure. In schizophrenia are found both alterations of certain microtubuleassociated proteins (MAP2 and MAP5) and alterations of certain neurotransmitter systems (dopaminergic and serotoninergic). Typical molecular finding is lowered $\mathrm{D}_{1}$ receptor activity in the brain cortex and increased $\mathrm{D}_{2}$ and $\mathrm{D}_{4}$ receptor activity in the limbic system. The 5-HT activity is responsible for the negative symptoms of schizophrenia that is why 5- $\mathrm{HT}_{2}$ antagonists are also used in the treatment. In Alzheimer's disease (AD) there is primary impairment of the axonal microtubule-associated protein tau, which is extensively phosphorylated. Hyperphosphorylated tau forms tangles and disrupts the cytoskeleton. In AD there is reported altered cholinergic neuromediator system. Typical GPCR induced psychiatric disorder is the drug addiction. All opiate receptors $\left(\mu . \kappa, \delta, \varepsilon\right.$ and N/OFQ) are $\mathrm{G}_{\mathrm{i} / \mathrm{o}}$ coupled and lead to inhibition of the adenylyl cyclase and drastic reorganization of the dendritic tree. The cytoskeletal disassembly and the dendritic loss are considered to correlate with the weight of the clinical symptoms in addiction. Cannabinoids like marijuana also act on $\mathrm{G}_{\mathrm{i} / \mathrm{o}}$ coupled receptors $\left(\mathrm{CB}_{1}\right.$ and $\left.\mathrm{CB}_{2}\right)$. In depression there is alteraion in the serotoninergic 5 -HT receptors. From the brief review of the molecular changes in the presented psychiatric disorders it is clear that the neural network theorists cannot provide satisfactory explanation for the huge variety of feelings evoked by different neurotransmitters if they just take into consideration the increased or decreased neuronal firing. The presented data without doubt points to subneuronal basis for conscious experience and mind.

\section{Neuronal electric activity and cytoskeleton}

We already have discussed that when neuromediator molecules are released in the synaptic cleft by the presynaptic terminal they bind to postsynaptic receptors. The GPCRs activation leads to slow biochemical organization of the neuron, while the ion channel activity leads to fast electric activity considered to correlate with conscious experience. Woolf $(1997,1999)$ as well as Woolf \& Hameroff (2001) suggested that the link between the electric encoded information and the cytoskeleton does not require special explanation but indeed the information is trasferred with the G-protein coupled biochemical cascades inducted by the neurotransmitters (acetylcholine, glutamate, serotonin) that activate phosphoinositidespecific phospholipase C (PI-PLC), PKC and $\mathrm{Ca}^{2+} /$ calmodulin activated kinase (CaMK II), which phosphorylate MAP2 molecules in dendrites that detach from microtubules. Even if we extend the model including the biochemical processes taking part in axons, the WoolfHameroff suggestion artificially neglects the role of the fast electric activity of the neurons in conscious processes and insists on the slow biochemical changes that once started have prolonged action (after-effects) and cannot be reversed so fast. If we remember Penfield's experiments the patient could experience one and the same event $\sim 60$ times without something is added or missed. And the most important: the effect last as long as electric stimulus is applied and disappears as soon as the electric current is stopped. Thus the makeor-break issue of the proposed model for conscious experience associated with activities taking part in the neuronal cytoskeleton depends on proving sensitivity of the cytoskeletal microtubules to the intraneuronal electric field. 
Georgiev (2003a) has performed computational study of the electromagnetic field strength in different neuronal compartments (dendrites, soma, axon) showing that the electric field intensity is $1-10 \mathrm{~V} / \mathrm{m}$ and could input information to the microtubules. The magnetic field strength is $10^{-10}-10^{-7} \mathrm{~T}$ and is suffocated by the Earth's magnetic field ruling out magnetic effects as input source of information. The calculated values for the electric field are questioning the validity of virtually all known models dealing with the microtubule interaction with the electromagnetic field. That is why Georgiev (2003a) proposed a novel model in which the highly acidic tubulin C-termini known as "tubulin tails" not only project from the microtubule surface and interact with MAPs and motor proteins, but are sensitive to the electric environment. The tubulin tail conformational status could be the clue towards solving this last problem and could provide us with deeper understanding of the cytoskeletal role in cognition.

\section{Exocytosis and neuromediator release}

Findings by Jack et al. (1981) inevitably imply an activation barrier, which hinders vesicular docking, opening, and releasing of transmitter molecules at the presynaptic membrane upon excitation by an incoming nerve impulse. Redman (1990) demonstrated in single hippocampal pyramidal cells that the process of exocytosis occurs only with probability generally much smaller than one upon each incoming impulse. Deconvolution analysis by Sayer et al. $(1989,1990)$ has shown that an impulse invading bouton evokes exocytosis (release of neuromediator molecules) with probability $0.2-0.3$. Considering the presented data it is safe to assume that only $1 / 4$ of the axonal terminals (boutons) release neuromediator under firing. This will produce complex geometric pattern of active $(\sim 25 \%)$ and silent $(\sim 75 \%)$ boutons that surely must be controlled by the conscious experience. The fact that $a$ single axon forms synapses with dendrites from hundreds of other neurons and the relatively small number of released vesicles makes the idea of their random (chaotic) release untenable i.e. it must be decided which neurons will be activated and which will not and this decision must be dependent on the current conscious experience!

One of the most interesting things about exocytosis is that the synaptic vesicles located in the presynaptic vesicular grid (hexagonal paracrystalline structure) of the axonal bouton manifest long-range correlations (Eccles, 1992; Beck \& Eccles, 1992). There are about 40 synaptic vesicles in the vesicular grid but never does more than one vesicle emit transmitter molecules into the synaptic cleft after stimulation by a nerve impulse. This certainly means that that the vesicles in the vesicular grid do not act independently but rather that immediately after one vesicle is triggered for releasing its content the interaction between them blocks further exocytosis. The paracrystalline structure of the presynaptic vesicular grid makes it possible to have long-range interactions between the constituents, as is well known from ordered quantum systems. Further Beck \& Eccles (1992) have calculated that the mass of the quasiparticle that promotes the exocytosis via tunneling is exactly that of a hydrogen atom. This supports the idea of vibrationally assisted tunneling as trigger for exocytosis as worked out by Georgiev (2002a). It is known that the pull of synaptic vesicles available for presynaptic membrane docking is regulated via synapsin I attachment to actin filaments. After releasing from the actin network different scaffold proteins regulate vesicle tethering, 
docking and fusion with the presynaptic membrane. The exocytotic machinery includes several families of SNARE proteins (VAMP/synaptobrevin, SNAP-25, synaptotagmin, syntaxin) and is regulated by additional SNARE master proteins. Detailed description of the mechanism of exocytosis and the proposed model with $\beta$-neurexin promotion of synaptotagmin-1 detachment from the SNARE complex under $\mathrm{Ca}^{2+}$ entry (axonal terminus excitation) could be found in Georgiev (2002a) that is why we will not go into details. What is important to note is that the vibrational multidimensional tunnelling needs classically allowed pathways in the conformational space and strongly relies upon "protein breathing" (that is protein thermal fluctuations). The process of neuromediator release is nothing but enzymatically driven catalysis and follows the experimentally established principles for H-tunnelling (Scrutton et al., 1999; Sutcliffe \& Scrutton, 2002). The proteins involved utilize the thermal energy for transient compression of the energy barrier of the reaction i.e. shortening of the tunnelling distance (Sutcliffe \& Scrutton, 2000).

\section{Synaptic switching}

In the mammalian brain, the contribution of intrasynaptic cellular adhesive molecules (CAMs) to synaptic plasticity has been studied mostly in the context of long-term potentiation (LTP). LTP can be induced at many different types of synapses throughout the brain but is best characterized in living brain-slice preparations of the hippocampus, a structure crucial for memory formation. When brief, high frequency trains of stimuli (tetanizing stimuli) are used to excite a neuron; a rapid-onset, short-lasting form of LTP (lasting 1-2 h) is induced that does not require protein synthesis. This form is called early (E)-LTP and very likely involves rapid changes in the strength of pre-existing synapses. By contrast, when multiple, widely spaced trains of high-frequency stimuli are used, both E-LTP and a subsequently developing, longer-lasting form of LTP (L-LTP) are induced, the latter lasting several hours to days or more. L-LTP requires gene transcription and protein synthesis and has been associated with growth of new dendritic spines and formation of new synapses. Each of the major families of CAMs has been shown to play a role in the induction or maintenance of E- and/or L-LTP. Georgiev (2003b) suggested that some of the conformational changes responsible for the E-LTP induced by enhanced electric activity are indeed responsible for the control of neurons involved in the conscious perception. It is known that all neurons do have background activity but nevertheless they do not produce any conscious image in resting state. That is the brain cortex has a lot of consciously realizable information stored, but not all this information is experienced all the time. If the mind is modelled by the activity of group of coherent neurons, then the conformational states of the $\beta$-neurexin-neuroligin-1 intrasynaptic protein bridges (also connecting the two neuronal cytoskeletons) could determine whether given neuron is switched in this coherent group or is switched off. For detailed exposition on CAMs role in brain (integrins, cadherins, NCAM, $\beta$-neurexin and neuroligin-1) and the associated impairment of memory formation in experimental studies when CAM function is interfered, the reader is referred to Georgiev (2003b). It is important to point out that the model involves proteins in the axo-dendritic synapses, because the split-brain experiments has clearly shown that axons are involved in integration of experience into single coherent "picture". 
In the next few paragraphs I would like to present brief description of the split-brain experiments, for which Sperry got Nobel Prize in 1981, and which clearly show that the suggested by Woolf \& Hameroff (2001) dendritic binding of conscious experience is insufficient to solve the "binding problem". The human nervous system has a bilateral symmetry most noticeable in the existence of the two cerebral hemispheres. The two halves of the brain, although exhibiting certain functional specializations, ordinarily work in an integrated manner to produce the conscious output of the nervous system, namely thought and action. Epilepsy is the general name given to a class of nervous system disorders involving convulsive activity of large numbers of nerve cells, and a classical surgical procedure in cases of severe epilepsy is section of the corpus callosum (commissurotomy), the large band of nerve fibres (axons) that serves as the primary connection between the two halves of the brain. Myers (1955) showed that when the cat had its optic chiasm and corpus callosum severed, two independent learning centers were established - one in each hemisphere of the cat's brain. If the cat had its right eye open and its left eye covered and learned to make a simple conditioned response, it was unable to make the same response when the right eye was covered and the left eye was open. It was as if the learning was unable to be communicated to the other side of the brain; thus, it was obvious that information available to one side remained off-limits to the other. Roger Sperry and Michael Gazzaniga (Gazzaniga \& Sperry, 1967; Sperry \& Gazzaniga, 1967; Sperry et al., 1969) began a series of studies of "split-brain" humans, patients who had the corpus callosum severed as a therapeutic procedure in patients with epilepsy, and the observations of these clinical patients have formed the basis for a number of significant ideas concerning brain function.

The World War II veteran (known in the scientific literature as W.J.) had undergone surgery to alleviate his epileptic seizures. After the surgery W.J. easily named and described colours, letters, and other information flashed briefly to the right side of his visual field; therefore, W.J.'s left hemisphere needed no help handling basic tasks requiring verbal responses. Then the scientists flashed items in W.J.'s left visual field and waited for the responses of his right hemisphere. As the anxious investigators looked on, W.J. acted as though he had suddenly gone blind. He insisted that he could not see bursts of light, boldface letters, or anything else presented to him. Yet his left hand, under the control of his right hemisphere, pushed down on a telegraph key each time a visual stimulus appeared, just as the scientists had instructed him to do.

In his Nobel lecture Sperry (1981) concluded that after commissurotomy "each of the disconnected hemispheres, not only the left, has its own higher gnostic functions. Each hemisphere in the lateralized testing procedures appeared to be using its own percepts, mental images, associations and ideas. As in the split-brain animal studies, each could be shown to have its own learning processes and its own separate chain of memories, all of course, essentially inaccessible to conscious experience of the other hemisphere". That is after commissurotomy the human brain hosts not one but two minds, clearly showing that any model relying $O N L Y$ on dendro-dendritic integration of conscious experience is inconsistent with the split-brain studies. 


\section{Picosecond protein dynamic timescale}

In recent paper Brunori et al. (1999) discussed the survival value of picosecond protein dynamics. Taking into account the experimental data obtained by overall reaction kinetics, single crystal X-ray diffraction, nanosecond laser photolysis and picosecond molecular dynamics simulations of sperm whale myoglobin $(\mathrm{Mb})$ mutant abbreviated with $\mathrm{Mb}-\mathrm{YQR}$, they have concluded that picosecond motions of the internal side chains in the active site of $\mathrm{Mb}$ control the overall rate of $\mathrm{O}_{2}$ disassembly. The picosecond fluctuations of the side chains either permit or obstruct access of $\mathrm{O}_{2}$ to cavities within the protein from which $\mathrm{O}_{2}$ can escape. Based on their finding Brunori et al. (1999) proposed that fast picosecond dynamic fluctuation have survival value not only in haemoproteins but possibly in all proteins.

The forces that govern atomic motions in proteins (macromolecules) include hydrogen bonding, electrostatic, and van der Waals interactions, as well as pseudo-forces arising from the hydrophobic effect (Zaccai, 2000). Although they are weak forces because their associated energies are similar to thermal energy at usual temperatures, they are responsible for protein-protein interactions and biological catalysis. Indeed all protein driven processes essential for life include induction of protein conformational changes with associated disruption and formation of noncovalent bonds that is why all proteins could be referred to as enzymes. By building on Pauling's assertion that any long-lived, chemically distinct interaction is a chemical bond, Purich (2001) redefines enzyme catalysis as the facilitated making and/or breaking of chemical bonds, not just of covalent bonds. He also introduces the term "energases" for certain class of enzymes that spend energy for biomolecular work or to induce conformational change in other proteins. Following Purich's definition and assuming that in protein-protein interactions there is "primed" energy that is released under the interaction, always one of the proteins will act as "energase" (i.e. will spend energy for inducing conformational change in the other protein). The term "primed" energy refers to accumulated energy in the protein conformational state gained by ATP or GTP hydrolysis in previous biochemical steps.

Taking into account that virtually all proteins possessing biologically important function could be referred to as "energases" and the new proposed mechanism for through-the-barrier enzyme catalysis (Scrutton et al., 1999; Sutcliffe \& Scrutton, 2000) we could be confident that the protein dynamic action in the cytoplasm depends on "thermal breathing" and proceeds via vibrationally assisted tunnelling. Here it should be noted that the electron tunnelling could be pure and does not depend on temperature, so not in all cases the tunnelling process must be vibrationally assisted. Indeed we have started from proposing the vibrationally assisted tunnelling as main paradigm, because in both cases (the law and the exception) there is quantum tunnelling through phase space. The older suggestion for "pure tunnelling" in biological media applied by Beck \& Eccles (1992) for exocytosis, lead the critics directly to the conclusion that because the temperature independence prophecy is not fulfilled all quantum effects in exocytosis are ruled out. For exposition of the WKB theory of multidimensional mixed tunnelling the reader is referred to Takada \& Nakamura (1994, 1995); details on vibrationally assisted tunnelling in exocytosis could be found in Georgiev (2002a). 


\section{Microtubule dynamic timescale}

Microtubule dynamic timescale coincides with the dynamic timescale of tubulin conformational transitions. Because in the brain most of the microtubules are stable, and usually it is considered that stable microtubules act as quantum computers, it is important to know how the microtubule could output information. Because the microtubules are the basic constituent of the neuronal cytoskeleton they act as scaffold for assembly of huge number of protein classes: microtubule-associated proteins (MAP1, MAP2, MAP5, tau), motor proteins (kinesin, dynein), microtubule severing proteins (Op18/stathmin, katanin), scaffold and bridging proteins (plakins, BPAG) etc. The tubulin conformational state is important and indeed exactly it is considered to represent a qubit. Microtubules act as orchestrators of the intraneuronal activity by the interaction of the individual tubulins with the associated MAPs, motor and scaffold proteins. That is why the microtubule functional dynamical timescale is in the order of 10 to 15 picoseconds as already discussed for enzymatic action.

Tubulin is heterodimer composed from $\alpha$ and $\beta$ subunit. Both subunits bind GTP, but only $\beta$-tubulin bound GTP is exchangeable (hence the GTP binding pocket is called E-site). Once integrated in the microtubule the E-site bound GTP is hydrolysed and converted to GDP. Only at the microtubule end there is a single layer non-hydrolysed GTP molecules called "cap" that prevents the microtubule from destabilization. Fujii \& Koizumi (1999) studied the associations of MAPs with tubulin. They found tubulin to undergo many posttranslational modifications at or near the carboxyl termini of the subunits. These C-termini are rich in acidic amino acids and have been shown to be involved in tubulin binding to MAPs. Specifically, they found the MAPs to bind $\alpha$-tubulin at amino acid sequences Lys430-Glu441 and $\beta$-tubulin at amino acid sequences Tyr 422-Gly 434. C-termini of $\alpha$ - and $\beta$-tubulin known as tubulin tails are rich in acidic aminoacid residues that is why the tubulin tails are highly flexible, mobile, susceptible to proteolysis, and exposed to the solvent. The tubulin $\mathrm{C}$-termini are prone to intense modification and regulation by different biochemical pathways inside neurons. Modifications such as acetylation, palmitoylation, phosphorylation and polyglutamylation are posttranslational modifications found on other proteins; others such as detyrosination and polyglycylation appear to be tubulin specific. Tyrosination-detyrosination is one of the major posttranslational modifications in which the C-terminal tyrosine residue in $\alpha$-tubulin is added or removed reversibly. Recent evidence indicates that detyrosination of tubulin can regulate interaction of MTs with vimentin intermediate filaments by a kinesindependent mechanism. The tubulin modifications polyglutamylation and polyglycylation involve the attachment of oligoglutamyl and oligoglycyl side chains of variable length to specific glutamate residues located near the $\mathrm{C}$-terminus of both $\alpha$ - and $\beta$-tubulin. Polyglutamylation represents the major posttranslational modification of axonal tubulin in neuronal cells, where it appears to regulate the differential interaction between MTs and MAPs. For instance, MAPs such as tau and kinesin exhibit optimal binding to tubulin modified by $\sim 3$ glutamyl residues, binding affinity decreasing with increased polyglutamyl chain length. In contrast, increasing polyglutamyl chain length does not appear to affect the binding affinity of MAP1A significantly. Considerable evidence suggests that there is an interaction between G-proteins and the cytoskeleton. G-proteins serve many functions involving the transfer of signals from cell surface 7-TM receptors to intracellular effector molecules and they may associate in cells directly with MTs. G-protein $\alpha$ subunits $\alpha_{\mathrm{il}}, \alpha_{\mathrm{s}}$, and 
$\alpha_{\mathrm{o}}$ are shown to activate the GTPase activity of tubulin, inhibit MT assembly, and accelerate MT dynamics. $\alpha_{i 1}, \alpha_{s}$, and $\alpha_{\mathrm{q}}$ bind to tubulin with high affinity. This binding appears to activate the G-proteins in association with a direct transfer of GTP from the E-site in tubulin to $\mathrm{G} \alpha$ (transactivation). In addition to activating $\mathrm{G} \alpha$, the association between $\mathrm{G} \alpha$ and tubulin induces a GTPase activity in tubulin similar to that seen after the self-association of tubulin dimers during the formation of a MT. Recent studies have also shown that G $\beta 1 \gamma 2$ binds to MTs and promotes MT assembly in vitro. Recent molecular studies have shown that free tubulin has and chaperone-like function (i.e. it helps other proteins to fold properly).

From the presented brief review of microtubule biology we could summarize: i) microtubules interact with the associated MAPs in manner defined by the conformational states of the individual tubulins, ii) tubulin could act as biological catalyst, so microtubule dynamic timescale is $10-15 \mathrm{ps}$, iii) tubulin/microtubules are intensively covalently modified by G-protein coupled cascades and could directly interact with $\mathrm{G} \alpha$ and $\beta \gamma$ subunits, iv) tubulin tails could be sensitive to the intraneuronal electric field inputting sensory information.

\section{Does cognition rely on millisecond process?}

In the current neural network models consciousness is linked to neural membrane firing and is considered to operate at the millisecond timescale. Curiously Jaynes (1976) argued that there must be gaps in consciousness due to the time it takes for neurons to fire or communicate through chemical neurotransmission. Dennett (1991) further suggested that the brain might fill in for gaps in consciousness, giving only the impression of continuity. Although this discontinuity of consciousness could be resolved there is no definitive evidence that millisecond process produces consciousness. The two seemingly most important arguments for millisecond timescale of consciousness are i) the millisecond timescale of conscious response when the subject is given certain task and ii) the subjective flow of time.

We already have seen that the first argument is not necessarily valid. The neural impulses reach the brain cortex in milliseconds and the motor response (motion, speech) is also achieved via neural impulses. The millisecond delay of the sensory impulses has biological importance because the impulses are not just transmitted but are also additionally processed on its way to the cortex by the relay spinal neurons and the thalamus, so that they pertain only biologically significant information. The information then is inputted from thalamus to the cortex by the thalamocortical projections. Also the brain cortex does not need to care about the precise control of every tiny step of the motor process. The output of the brain cortex is by the pyramidal neurons in the motor cortex whose axons form the pyramidal pathway. The axonal terminals of the cortical neurons form synapses with interneurons in the spinal cord that inhibit the $\alpha$-motoneurons. That is why the pyramidal pathway has inhibitory action on $\alpha$-motoneurons regardless of the fact that the released neuromediator (glutamate) is excitatory. Indeed in the motor control participate also nucleus ruber and cerebellum. We could roughly say that the cerebellum does most of the informational processing needed and the brain cortex is only navigating the whole system. The situation is the same with a human being working on its personal computer. It enters commands, receives ready output and then 
enters new commands without necessarily knowing how exactly the computer performs the computation. The communication between the brain cortex and the environment is achieved via electric impulses in milliseconds, possibly because this was the optimal biologically feasible solution, but this does not imply lower limit of the duration of the computational steps of the cortex i.e. it is known only that each step lasts no longer than the response time (milliseconds). So we could conclude that the conscious response time only establishes upper limit of the duration of the computational steps performed by the cortex and cannot be considered as proof that millisecond process is responsible for conscious experience.

In this paragraph we will focus our attention on the second argument and try to find out could the subjective flow of time be considered as proof for the millisecond based consciousness hypothesis. It could be intuitively expected that if mind operates in picosecond timescale, for example, the milliseconds (whose duration is $10^{9}$ times longer) would be subjectively experienced as decades or even centuries. Almost all neural network theorists modelling mind as reverberatory circuits of travelling neural impulses maybe intuitively share this view. However, as we will explain in detail, it is disproved by the experimental data. First, it would be biologically unfeasible to have such prolonged experience of a millisecond, because brain communicates with the environment in milliseconds, and second, experiments show that consciousness operates beyond our ability to construct time from reading out time labels produced by internal timekeeper mechanism ticking in wide range from milliseconds to seconds (Rao et al., 2001). Observed rare cases in the clinical practice of patients with time agnosia clearly show that consciousness is possible without perception of time and that for these patients apparent dynamics of observed objects do not produce feeling of "time flow", as intuitively expected. Thus if our mind operates at $100 \mathrm{GHz}$ (dynamical timescale of 10 picoseconds), every single "occasion of consciousness" experienced as "present" could be result from $10^{10}-10^{11}$ conscious steps sharing the same "time label". In the next chapter we will try to present the biological basis for time perception and the emergence of subjective time from information i.e. from reading "time labels" produced by the basal ganglia.

\section{Basal ganglia as internal timekeeper}

Rao et al. (2001) have performed complex experimental study using fMRI and have been able to locate the "internal clock" in brain responsible for producing time labels that could be used to calculate the time interval between two events and construct the experienced flow of time by the brain cortex (here is appropriate to note that constructivist theory of mind suggests that brain cortex constructs the experience from the sensory input (Nakamura \& Chapman, 1999) i.e. the cortex processes and converts the electrical input into conscious states).

Most theories of prospective timing embody an internal timekeeper, attention and memory. A clock metaphor is used to describe the timekeeper mechanism, which represents subjective time through the accumulation or readout of pulses, possibly generated by oscillators. Our perception of time, however, is intimately related to the level of attention given to the passage of time. When attention is diverted, a systematic shortening of subjective duration occurs, implying that pulses from the timekeeper may be lost. Attention may also mediate the 
flexible starting and stopping of pulses from the timekeeper, which enables anticipation of predictable events. Hence, a representation of subjective time emerges from the interplay between timekeeping and attention mechanisms. This representation is then passed on to working memory, a short-term repository where interval representations are maintained and manipulated in accord with current goals (for example, comparing two intervals of time). Working memory functions can therefore alter stored representations of time as well. The combination of these different component processes gives rise to the subjective perception of time, although the relative contribution of each might differ depending on the interval duration or the cognitive demands of timing events. Rao et al. (2001) provide compelling evidence for the involvement of the basal ganglia in formulating representations of time. Activation in the right putamen and caudate were uniquely associated with encoding time intervals. These results corroborate studies in Parkinson's disease showing that dopaminergic treatment improves motor timing (O’Boyle et al., 1996; Pastor et al., 1992) and time perception (Malapani et al., 1998). Pharmacological challenges in animals also suggest that dopaminergic antagonists and agonists respectively slow down and speed up timing operations (Maricq \& Church, 1983; Meck, 1986). The basal ganglia are involved in timing a wide range of intervals, from milliseconds to seconds. Collectively, these results implicate striatal dopaminergic neurotransmission in hypothetical internal timekeeping mechanisms.

The basal ganglia consist of striatum, substantia nigra and subthalamic nucleus. Striatum includes: neostriatum, paleostriatum and archistriatum. Neostriatum is derivative of the telencephalon and consists of caudate nucleus, the putamen and nucleus accumbens. Paleostriatum is derivative of the diencephalons and is represented by globus pallidus consisting of 2 parts: lateral (GPl) and medial (GPm). Archistriatum is division of the telencephalon and is represented by the amygdaloid nuclear complex. Substantia nigra is nucleus of the mesencephalon, however it is considered by many to be part of the basal ganglia because of its rich connections with the striatum. Substantia nigra has to parts: reticulata $(\mathrm{SNr})$ and compacta $(\mathrm{SNc})$. Subthalamic nucleus is derivative of the diencephalon. All the presented nuclei form extremely complex network forming closed loops between the cortex, basal ganglia, thalamus and cerebellum.

The neuroanatomical bridge for basal ganglia-cortical interactions is the thalamus, which was activated early during the encoding of intervals, along with two cortical regions, suggesting they work together in formulating representations of time. Coupled activation in the right inferior parietal cortex (BA 40) may suggest an interdependent role of this region in attention, which theoretically regulates the timekeeping mechanism. Neurological patients with right but not left inferior parietal damage show time perception deficits that correlate with impairments in switching attention (Harrington et al., 1998). The perception of time also relies on stored representations of intervals in working memory. During time perception, activation was observed in regions commonly associated with temporary storage functions, including the bilateral premotor (BA 6) and right DLPF cortex (BA 9, 10, 46) (GoldmanRakic, 1996; Smith \& Jonides, 1999; Cohen et al., 1997). Right DLPF activation was also unique to performing time discriminations. This corroborates with the finding that damage to these same regions in the right, but not left, hemisphere produces time perception deficits (Harrington et al., 1998). The reciprocal interactions among these specialized systems give rise to our perception of current time. The results are in agreement with converging avenues 
of research implicating a perceptual system in which the basal ganglia act as a timekeeper that is tightly coupled with an attention system in the right inferior parietal cortex. This right hemisphere bias for the encoding of temporal information is in agreement with converging focal lesion and electrophysiological research in humans.

\section{Time agnosia and acalculia}

Here I would like to present-in-brief a case from my clinical practice of a 40 -years old woman that had vascular incident with focal lesions in the cortex. She had interesting form of time agnosia associated with acalculia (inability to complete simple arithmetical problems, usually associated with injuries in the region of the angular gyrus (BA 39) or supramarginal gyrus (BA 40) or of the dominant (left) cerebral hemisphere). When asked to assess the time passed from event happened early in the day, she was not able to do so. Although the time was 10 a.m. she was not able to determine when she had breakfast, nor had idea that the day has duration. She had no idea what season it is, nor was able to report how the seasons are ordered. The strangest was that when asked to summate two different numbers e.g. $3+5$ she was not able to perform the task. Even the most elementary $1+2$ was unsolvable problem. Surprisingly she was able to summate two alike numbers e.g. $2+2$ or $5+5$ providing answers " 4 " and "10". Also there were no problems with multiplication. When I asked her to multiply $3 \times 7$ I unexpectedly received immediate reply " 21 ". Then I again tried with $1+2$ without success. Further I helped her with the tasks $1+1$ and $2+2$ and when I received the right answers " 2 " and " 4 " I suggested that $1+2$ must be number between the numbers 2 and 4 . For pity the answer 3 as if did not exist. I concluded that it is not because it is odd, because I had already received answer " 21 ", rather she was not able to order events, numbers etc. The summation of two alike numbers was preserved possibly because it could be performed via multiplication times 2 but I cannot be sure that she actually did that, nor I can explain why multiplication was preserved. After seeing her embarrassment that she cannot answer my questions, I decided to change the topic asking her about her previous job and her family. She explained that she was accountant (never had before problems with calculations) and that she is happy for the great support from her family in helping her to overcome the disease. I have found no other heavy psychiatric disturbances that lead me to the conclusion that really the time as if is not flowing for her. The examination showed that "time" and "addition of two different" numbers have no meaning for the patient, or in other words they are not consciously realizable options. The data corroborate the idea that time is constructed by information ("time labels") and that the subjective time flow is not consequence of observing moving or changing objects but is constructed by specialized structures. What is interesting is that my observation suggests that subjective time could be intimately linked to mathematical skills (and more specifically with ordering) and that the development of mathematical skills in human evolution (mathematics is considered to be typical "human acquirement") was accompanied by changes in the way we percept time. Indeed psychophysiological studies performed by Ernst Pöppel and co-workers (Pöppel, 1970; 1971; 1978; 1988; 1989a; 1989b; 1994; 1995; 1996; 1997; Pöppel \& Logothetis, 1986; Kanabus et al., 2002; Podvigin et al., 2003) succeeded to measure the duration of the minimal "quantum" of subjective time and revealed that the left temporal cortex is crucial for determining the time order of events. 


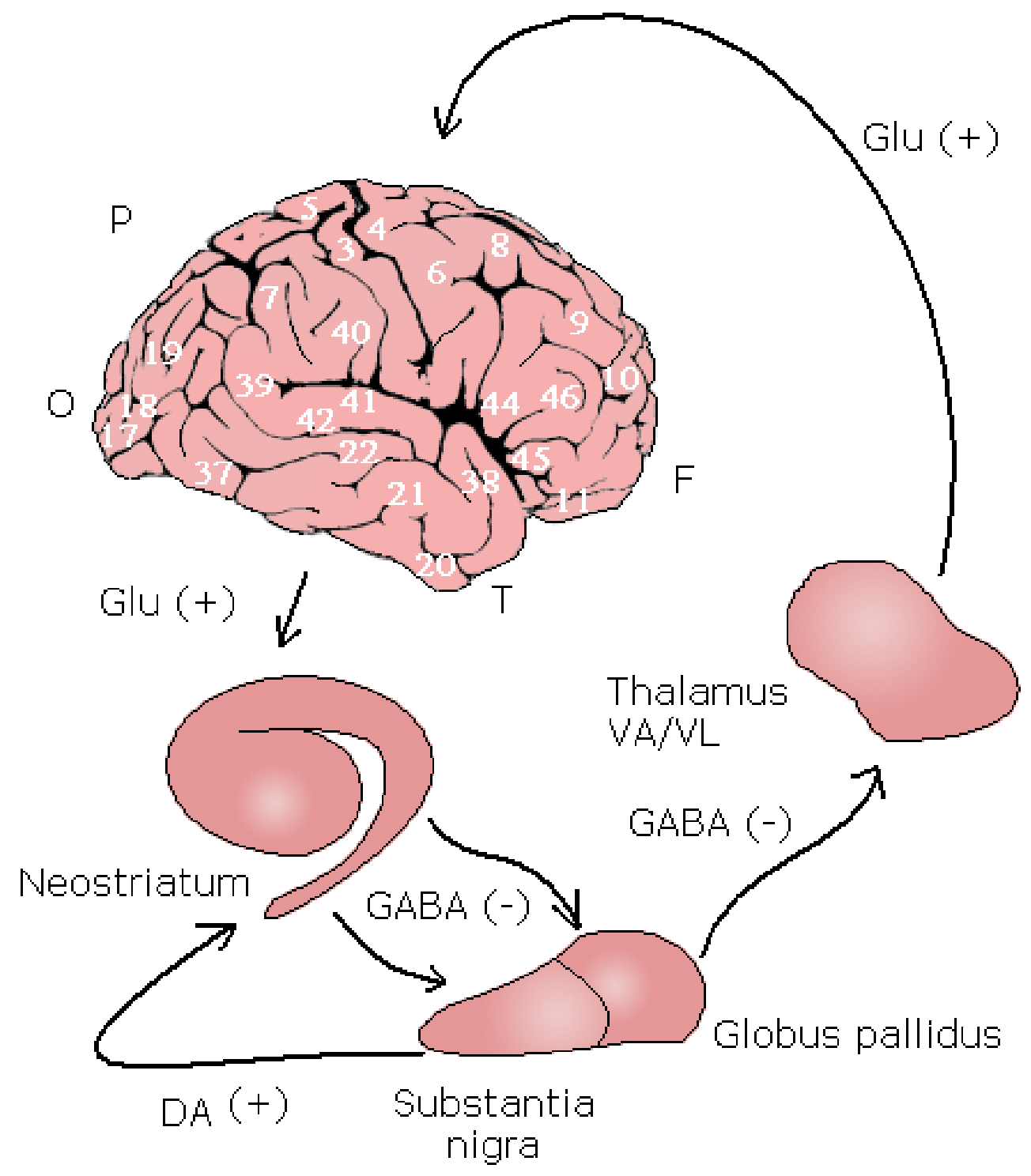

FIG.4 This schematic diagram of the principal path through the basal ganglia. Note that the system forms a loop. Information originates from nearly the entire cerebral cortex. After passing through the basal ganglia and thalamus, the modified information is returned primarily to the specific motor areas of the cerebral cortex. The principal connections of the basal ganglia are indicated by arrows and the neurotransmitters are indicated along with their apparent action: facilitation (+) or inhibition (-). The substantia nigra and the globus pallidus are functionally considered to be the same nucleus. This is similar to the situation with the caudate nucleus and putamen, which are indicated here simply as neostriatum. Basic neurotransmitters: Glu, glutamate; GABA, $\gamma$-amino butyric acid; DA, dopamine. Abbreviations: F, frontal lobe; $O$, occipital lobe; $P$, parietal lobe; $T$, temporal lobe, Brodmann areas are presented with numbers. 


\section{Subjective time quanta}

Rao et al. (2001) identified right basal ganglia as internal timekeeper that is responsible for estimation and comparison of different time intervals and therefore is intimately linked with the production of the subjective time flow. The subjective determination of the time order of events however, although intimately linked with the experience that time is going on, relies on more complex computational mechanism including the left temporal cortex. A number of experimental studies have provided evidence that the minimal inter-stimulus-interval (ISI) between two successive stimuli for correctly reporting their temporal order is about $20-40 \mathrm{~ms}$ (Hirsh \& Sherrick, 1961; Pöppel, 1997). This order threshold appears to be remarkably invariant for auditory, visual, tactile and two-modality stimuli in normal subjects (Hirsh \& Sherrick, 1961; Swisher \& Hirsh, 1972). Such a perceptual phenomenon is probably due to a central mechanism responsible for temporal ordering, which is independent of the sensory stimulus itself, the temporal cortex of the left hemisphere probably being responsible for temporal order judgement (Efron, 1963a; 1963b; Tallal et al., 1998; von Steinbüchel et al., 1999a; 1999b). Kanabus et al. (2002) investigated the ability to perceive temporal order for pairs of auditory and visual stimuli in 12 volunteers. They were asked to make judgements about the order of presented stimuli by pressing two response buttons in a certain order. The performance on auditory and visual tests was studied in relation to the ISI, which varied from 5 to $500 \mathrm{~ms}$. In general, the level of performance was similar for the two modalities and the criterion of $75 \%$ of correct responses was reached at ISI longer than $40 \mathrm{~ms}$, independently of the modality.

What is particularly interesting is that the minimal ISI that allows for discrimination of two consecutive stimuli as two (not merging them into one) is much shorter than the ISI needed for determination of time order. In visual perception two flashes were perceived as a single flash for intervals shorter than about 100 milliseconds for rods (Oya, 1997) and shorter than 10 to $15 \mathrm{~ms}$ for cones (Kalloniatis \& Luu, 2003). Indeed these are the integration times of rods and cones, respectively. Physiological experiments have shown that if two flashes are presented with ISI lasting longer than $40 \mathrm{~ms}$ their time order can be determined. If the ISI is in the interval $10-40 \mathrm{~ms}$, then the time order of the two consecutive flashes cannot be determined (or they seem to be simultaneous). If the ISI is beyond $10 \mathrm{~ms}$ the two consecutive flashes from a single source are merged into one single flash. Merging of two consecutive flashes into a single flash is explained by the processing of the visual information via graded potentials in the retina (for details see Georgiev, 2002b); and the inability to determine the time order for ISI shorter than $40 \mathrm{~ms}$ is explained as with the duration of the oscillatory period of the internal timekeeper in brain. If the brain cortex however operates at picosecond timescale as suggested in the current paper, then direct or transcranial electrical stimulation should show that the minimal ISI, which allows for discrimination of two consecutive stimuli applied directly to the cortex, lasts shorter than a millisecond. Thus the presented here idea no matter that is theoretically and experimentally supported could be further tested. 


\section{Microtubules as quantum system}

Tegmark (2000) proposes convenient classification of the physical systems based on three basic criteria (timescales): $\tau_{\mathrm{dyn}}$ - dynamical timescale of the system, $\tau_{\text {diss }}$ - dissipation time and $\tau_{\mathrm{dec}}-$ decoherence time. The dynamical timescale of a system is defined as that which is characteristic of its internal dynamics. For a planetary system or an atom, $\tau_{\mathrm{dyn}}$ would be the orbital frequency. The dissipation timescale $\tau_{\text {diss }}$ is defined as the time it would take to lose half of the initial excess energy above the thermal equilibrium. The third timescale is intimately linked to a purely quantum phenomenon called decoherence. Quantum decoherence is mathematically identified through either the diagonalisation of a density matrix $\rho_{\mathrm{i}}$, or the suppression of interference phases or the ability to adequately model a quantum system by a classical stochastic process. The density matrix of the system becomes rapidly (approximately) diagonal in a given basis. The basis in which the density matrix is diagonalised is called the pointer basis and the timescale after which this diagonalisation has occurred is known as decoherence time $\tau_{\text {dec }}$ (Anastopoulos, 2001). This effect destroys the ability to observe long-range quantum superpositions within the subsystems. The qualitative behaviour of a system depends on the ratio of these timescales. If $\tau_{\mathrm{dyn}}<\tau_{\mathrm{dec}}$ we are dealing with true quantum system, since its superpositions can persist long enough to be dynamically important. If $\tau_{\text {dyn }}>>\tau_{\text {diss }}$ it is hardly meaningful to view the system as independent system at all, since its internal forces are so week that they are dwarfed by the effects of the surroundings. In the intermediate case where $\tau_{\text {dec }}<<\tau_{\text {dyn }}<\tau_{\text {diss }}$ we have familiar classical system.

In attempt to disprove the quantum mind hypothesis Tegmark (2000) calculated the decoherence time of microtubule travelling kinks (solitons) concluding that microtubules are not quantum system. The error however is that Tegmark compares decoherence timescale of microtubule travelling solitons (kinks) with the dynamical timescale of membrane firing, something that is not biologically acceptable. Virtually he compares timescales of two different systems A and B and derives conclusion about A.

We have shown that microtubules act as orchestrators of intraneuronal activity via the actual conformational states of the individual tubulins. The calculated by Tegmark decoherence time of $10^{-13} \mathrm{~s}$ compared to the $10^{-11} \mathrm{~s}$ tubulin/microtubule dynamical timescale cannot rule out quantum effects. As Tegmark (2000) noted, the decoherence time could be increased with 1-2 orders of magnitude if we change the parameters in the equation: mainly the charge of the kink that is in superposition and the separation in space of the superposed kink. Also if we consider tubulin tail conformational superposition (not superposition of soliton) for the superposition separation we will have part of the nanometer (not extension of several tubulin lengths as Tegmark assumed). I would like to note that the established dynamic timescale of $10-15$ ps does not necessarily prove that microtubules act as quantum system, but it suggests new possibilities for theoretical modelling of microtubules. What about the resulting fast 100 $\mathrm{GHz}$ processing it is fully compatible with neurobiology and psychophysiology. 


\section{Could we process information faster then $100 \mathrm{GHz}$ ?}

In this short paragraph I would like to answer one very important question, namely: "Is the way open for searching the quantum mind down to the Planck's scale? After all we have seen that consciousness operates beyond the timescale for discerning time intervals and there won't be any psychophysiological paradox if we decrease the dynamic timescale?" Although the presented paper could be met by the Q-mind advocates with enthusiasm I should warn them that the proposed model is based on established experimental facts that impose certain limits. Tubulin catalytic action (as well as the control of exocytosis) is proposed to depend on the thermal protein breathing. Indeed the vibrationally assisted tunnelling utilizes the thermal energy either to promote or to suppress given process, that is why the dynamic timescale of conscious processes is pushed to the theoretical limit. Recently proposed models of boson condensation as mind process (with $10^{-14} \mathrm{~s}$ dynamics) cannot provide any biological function taking part at this timescale that is why I think the $100 \mathrm{GHz}$ conscious informational processing is the fastest possible.

\section{What makes the brain cortex unique?}

We have already discussed the differentiation of neurons in the brain cortex, and have described the characteristic morphology of typical cortical neurons. Although there is different morphology of the neurons presented in different Brodmann areas after all there must be something common that makes the cortex conscious. Could this be result from special protein (cytoskeletal) organization/dynamics?

The cell biologists are confident that the morphology is regulated via selective protein expression during development and the current paradigm holds that this is achieved via selective gene control. This means that certain genes are active in given period of development then they are turned off and another set of genes are activated. The signals for different gene switching on/off are coming from the cytoplasm that responds to extra-cellular stimuli. Indeed the cell nucleus (genome) contains all the "wisdom" that the cell has from its ancestors. However the living entity is the cell itself and it "learns" in order to adapt and survive. That is why we have considered that consciousness could be linked to neuronal cytoskeletal activity and have provided evidence that the neuronal cytoskeleton integrates all the incoming information and itself is capable to output information. In favour of the current protein mediated consciousness model against the neural network paradigm claiming that consciousness is electric field (impulses) is even the mere fact that what differs us from other animal species is the aminoacid sequence of our proteins.

Although we have only $1,23 \%$ difference in the genome compared to chimpanzee, when compared the brain proteome in humans and chimpanzee the difference raises up. Enard et al. (2002) observed a 5.5-fold acceleration of the rate of change in gene expression levels in human brains compared to chimpanzees. The rate of change in gene expression for blood and liver were measured $1.0 \%$ and $1.3 \%$ respectively. Also it is interesting that the proteome analysis of brain tissues showed that the quantitative differences in protein levels were six times greater than qualitative changes in the human brain. The study of the brain cortex 
transcriptome (mRNA transcripted) and proteome (proteins synthesized) in humans comparatively to the transcriptome and proteome of other animals will further provide us with insight which proteins are essential for consciousness (make the cortex so special) and/or which proteins make us humans.

\section{Acknowledgements}

I would like to thank to Stephen M. Rao (Department of Neurology; Cell Biology, Neurobiology and Anatomy, Medical College of Wisconsin) for providing materials of his work on the basal ganglia function as internal timekeeper in brain.

Special thanks to Huping Hu (Biophysics Consulting Group, 25 Lubber Street, Stony Brook, NY 11790) for the thoughtful discussion at the poster session (17 March 2003) of Quantum Mind II Conference held in Tucson, Arizona.

\section{References}

1. Anastopoulos, C. (2001). Frequently asked questions about decoherence. quant$\mathrm{ph} / 0011123$

2. Beck, F. \& Eccles, J.C. (1992). Quantum aspects of brain activity and the role of consciousness. PNAS 89: 11357-11361.

3. Brunori, M., Cutruzzola, F., Savino, C., Travaglini-Allocatelli, C., Vallone, B. \& Gibson, Q.H. (1999) Does picosecond protein dynamics have survival value? TiBS 24: 253-255.

4. Cohen, J. et al. (1997). Temporal dynamics of brain activation during a working memory task. Nature 386: 604-607.

5. Dennett, D.C. (1991). Consciousness explained. Boston: Little, Brown.

6. Dobelle, W.H. (2000). Artificial vision for the blind by connecting a television camera to the visual cortex. ASAIO Journal 2000; 46:3-9.

7. Eccles J.C. (1992). Evolution of consciousness. PNAS 89: 7320-7324.

8. Efron, R. (1963a). The effect of handedness on the perception of simultaneity and temporal order. Brain 86: 261-284.

9. Efron, R. (1963b). Temporal perception, aphasia and deja vu. Brain 86: 403-423.

10. Enard, W., Khaitovich, P., Klose, J. et al. (2002). Intra-and interspecific variation in primate gene expression patterns. Science 296: 340-343.

11. Fujii T, \& Koizumi Y. (1999). Identification of the binding region of basic calponin on $\alpha$ and $\beta$ tubulins. J Biochem (Tokyo) 125: 869-875.

12. Gazzaniga, M.S. \& Sperry, R.W. (1967). Language after section of the cerebral commissures. Brain, 90, (I), 131-148.

13. Georgiev, D. (2002a). The causal consciousness: $\beta$-neurexin promotes neuromediator release via vibrational multidimensional tunneling. quant-ph/0210102 
14. Georgiev, D. (2002b). Where do the photons collapse - in the retina or in the brain cortex? quant-ph/0208053

15. Georgiev, D. (2003a). Electric and magnetic fields inside neurons and their impact upon the cytoskeletal microtubules. CogPrints ID 00003190.

16. Georgiev, D. (2003b). Solving the binding problem: cellular adhesive molecules and their control of the cortical quantum entangled network. CogPrints ID 00002923.

17. Goldman-Rakic, P.S. (1996). Regional and cellular fractionation of working memory. PNAS 93: 13473-13480.

18. Harrington, D.L., Haaland, K.Y. \& Knight, R.T. (1998). Cortical networks underlying mechanisms of time perception. J. Neurosci. 18: 1085-1095.

19. Hirsh, I.J. \& Sherrick, C.E. (1961). Perceived order in different sense modalities. J. Exp. Psych. 62: 423-432.

20. Jack, J.J.B., Redman, S.J. \& Wong, K. (1981). The components of synaptic potentials evoked in a cat spinal motoneurons by impulses in single group Ia afferents. J. Physiol. 321: 65-96.

21. Jaynes, J. (1976). The origin of consciousness in the breakdown of the bicameral mind. Boston: Houghton Mifflin.

22. Kalloniatis, M. \& Luu, C. (2003). Psychophysics of vision. http://webvision.med.utah.edu/

23. Kanabus, M., Szelag, E., Rojek, E. \& Pöppel, E. (2002). Temporal order judgement for auditory and visual stimuli. Acta Neurobiol. Exp. 62: 263-270.

24. Malapani, C. et al. (1998). Coupled temporal memories in Parkinson's disease: a dopamine-related dysfunction. J. Cogn. Neurosci. 10: 316-331.

25. Maricq, A.V. \& Church, R.M. (1983). The differential effects of haloperidol and methamphetamine on time estimation in the rat. Psychopharmacology 79: 10-15.

26. Meck, W.H. (1986). Affinity for the dopamine D2 receptor predicts neuroleptic potency in decreasing the speed of an internal clock. Pharmacol. Biochem. Behav. 25: 1185-1189.

27. Myers, R.E. (1955). Interocular transfer of pattern discrimination in cats following section of crossed optic fibers. J. Comp. Physiol. Psychol., 48.

28. Nakamura, Y. \& Chapman, C.R. (1999). Constructing pain: how pain hurts? In: No matter, never mind. Proceedings of Towards a science of consciousness: fundamental approaches (Tokyo '99). Yasue, K., Jibu, M. \& Della Senta, T. (eds.) pp. 193-206.

29. Nieuwenhuys, R. (1994). The neocortex. An overview of its evolutionary development, structural organization and synaptology. Anat Embryol (Berl) 190 (4): 307-337.

30. O'Boyle, D.J., Freeman, J.S. \& Cody, F.W.J. (1996). The accuracy and precision of timing of self-paced, repetitive movements in subjects with Parkinson's disease. Brain 119: $51-70$.

31. Oya, S. (1997). Effective luminous intensity of two successive flashing lights. Journal of Light and Visual Environment 21: 6-10.

32. Pastor, M.A., Artieda, J., Jahanshahi, M. \& Obeso, J.A. (1992). Performance of repetitive wrist movements in Parkinson's disease. Brain 115: 875-891.

33. Penfield, W.G. (1954a). Some observations of the functional organization of the human brain. Proc. Am. Phil. Soc. 98 (5), 293-297. 
34. Penfield, W.G. (1954b). Studies of the cerebral cortex of man: a review and an interpretation. In: Brain mechanisms and consciousness. Delafresnay, J.F. (ed.). Oxford: Blackwell Scientific Publications. pp. 284-309.

35. Penfield, W.G. (1955). The role of the temporal cortex in certain psychical phenomena. J. Ment. Sci. 101, 451-465.

36. Penfield, W.G. (1969). Epilepsy, neurophysiology and some brain mechanisms related to consciousness. In: Basic mechanisms of the epilepsies. Jasper, W. \& Pope, L. (eds.). Brown \& Co., Boston.

37. Penfield, W.G. \& Perot, P. (1963). The brain's record of auditory and visual experience: a final summary and discussion. Brain 86: 595-696.

38. Penrose, R. (1989). The emperor's new mind. Oxford, Oxford University Press.

39. Penrose, R. (1997). In: The large, the small and the human mind. M. Longair (ed.). Cambridge, Cambridge University Press.

40. Podvigin, N.P., Bagaeva, T.V., Podvigina, D.N. \& Pöppel, E. (2003). On possible neurophysiological basis of the process of short time interval estimation. In Proceedings: Time, cognition, thinking. Strzekecino, Poland, 19-23 July.

41. Purich, D.L. (2001). Enzyme catalysis: a new definition accounting for noncovalent substrate- and product-like states. TiBS 26: 417-421.

42. $\quad$ Pöppel, E. (1970). Excitability cycles in central intermittency. Psychol. Forschung 34: $1-9$.

43. Pöppel, E. (1971). Oscillations as possible basis for time perception. Studium Generale 24: 85-107.

44. Pöppel, E. (1978). Time perception. In: Handbook of sensory physiology. Held, R., Leibowitz, H. \& Teuber, H.L. (eds.). Berlin, Springer-Verlag, pp. 713-729.

45. Pöppel, E. (1988). Mindworks. Time and conscious experience. Harcout Brace Jovanovich, Boston.

46. Pöppel, E. (1989a). The measurement of music and the cerebral clock: a new theory. Leonardo 22: 83-89.

47. Pöppel, E. (1989b). Taxonomy of the subjective: an evolutionary perspective. In: Neuropsychology of visual perception. Jason W. Brown, M.D. (ed.). pp. 219-232

48. $\quad$ Pöppel, E. (1994). Temporal mechanisms in perception. Int. Rev. Neurobiol. 37: 185202.

49. Pöppel, E. (1995). Time perception: problems of representation and processing. In: The handbook of brain theory and neural networks. Arbib, M.A. (ed), pp. 987-990

50. Pöppel, E. (1996). Reconstruction of subjective time on the basis of hierarchically organized processing system. In: Time, internal clocks and movement. Pastor, M.A. \& Artieda, J. (eds). pp. 165-185

51. Pöppel, E. (1997). A hierarchical model of temporal perception. TiCS 1: 56-61.

52. Pöppel, E. \& Logothetis, N. (1986). Neuronal oscillations in the brain. Discontinuous initiations of pursuit eye movements indicate a 30-Hz temporal framework for visual information processing. Naturwissenschaften 73: 267-268.

53. Rao, S.M., Mayer, A.R. \& Harrington, D.L. (2001). The evolution of brain activation during temporal processing. Nature Neuroscience 4: 317-323.

54. Redman, S. J. (1990). Quantal analysis of synaptic potentials in neurons of the central nervous system. Physiol. Rev. 70: 165-198. 
55. Sayer, R.J., Redman, S.J. \& Andersen, P. (1989). Amplitude fluctuations in small EPSPs recorded from CA1 pyramidal cells in the guinea pig hippocampal slice. J. Neurosci. 9: 845-850.

56. Sayer, R.J., Friedlander, M.J. \& Redman S.J. (1990). The time course and amplitude of EPSPs evoked at synapses between pairs of CA3/CA1 neurons in the hippocampal slice. J. Neurosci. 10: 626-636.

57. Scrutton, N.S., Basran, J. \& Sutcliffe, M.J. (1999). New insights into enzyme catalysis: Ground state tunnelling driven by protein dynamics. Eur. J. Biochem. 264: 666-671.

58. Smith, E. \& Jonides, J. (1999). Storage and executive processes in the frontal lobes. Science 283: 1657-1661.

59. Sperry, R.W. (1981). Some effects of disconnecting the cerebral hemispheres. Nobel lecture

60. Sperry, R.W. \& Gazzaniga, M.S. (1967). Language following disconnection of the hemispheres. In: Brain mechanisms underlying speech and language. Millikan, C.H. \& Darley, F.L. (eds.). New York: Grune \& Stratton, Inc., 177-184.

61. Sperry, R.W., Gazzaniga, M.S. \& Bogen, J.E. (1969). Interhemispheric relationships: the neocortical commissures; syndromes of hemisphere disconnection. In: Handbook of clinical neurology. Amsterdam. Vinken, P.J. \& Bruyn, G.W. (eds.). North-Holland Publishing Company, 4, 177-184.

62. Sutcliffe, M.J. \& Scrutton, N.S. (2000). Enzymology takes a quantum leap forward. Philos. Trans. R. Soc. London Ser. A 358: 367-386.

63. Sutcliffe, M.J. \& Scrutton, N.S. (2000). Enzyme catalysis: over-the-barrier or through-the-barrier? TiBS 25: 405-408.

64. Sutcliffe, M.J. \& Scrutton, N.S. (2002). A new conceptual framework for enzyme catalysis: Hydrogen tunneling coupled to enzyme dynamics in flavoprotein and quinoprotein enzymes. Eur. J. Biochem. 269: 3096-3102.

65. Swisher, L. \& Hirsh, I.J. (1972). Brain damage and the ordering of two temporally successive stimuli. Neuropsychologia 10: 137-152.

66. Takada, S. \& Nakamura, H. (1994). Wentzel-Kramer-Brillouin theory of multidimensional tunneling: General theory for energy splitting. J. Chem. Phys. 100: 98-113. http://theory.chem.sci.kobe-u.ac.jp/PDF/Takada 94JCP.pdf

67. Takada, S. \& Nakamura, H. (1995). Effects of vibrational excitation on multidimensional tunneling: General study and proton tunneling in tropolone. J. Chem. Phys. 102: 3977-3992. http://theory.chem.sci.kobe-u.ac.jp/PDF/Takada 95JCP.pdf

68. Tallal, P., Merzenich, M.M., Miller, S. \& Jenkins, W. (1998). Language learning impairments: integrating basic science, technology, and remediation. Exp. Brain Res. 123: 210-219.

69. Tegmark, M. (2000). The importance of quantum decoherence in brain processes. Phys. Rev. E 61: 4194-4206, quant-ph/9907009

70. von Steinbüchel, N., Wittmann, M. \& Szelag, E. (1999a). Temporal constraints of perceiving, generating, and integrating information: clinical indications. Restor. Neurol. Neurosci. 14: 167-182.

71. von Steinbüchel, N., Wittmann, M., Strasburger, H. \& Szelag, E. (1999b). Auditory temporal order judgement is impaired in patients with cortical lesions in posterior regions of the left hemisphere. Neurosci. Lett. 264: 168-171. 
72. Woolf, N.J. (1997). A possible role for cholinergic neurons of the basal forebrain and pontomesencephalon in consciousness. Consciousness \& Cognition 6: 574-596.

73. Woolf, N.J. (1999). Dendritic encoding: an alternative to temporal synaptic coding of conscious experience. Consciousness \& Cognition 8: 447-454.

74. Woolf, N.J. \& Hameroff, S. (2001). Quantum approach to visual consciousness. TiCS 5: 472-478.

75. Zaccai, G. (2000). How Soft Is a Protein? A Protein Dynamics Force Constant Measured by Neutron Scattering. Science 288: 1604-1607. 